\title{
Linx
}

Revue des linguistes de l'université Paris X Nanterre

40 | 1999

Le statut d'unité lexicale

\section{L'unité lexicale dans la perspective harrissienne}

Lexical Unity in a Harrisian Perspective

\section{Danielle Leeman}

\section{OpenEdition}

Journals

Édition électronique

URL : http://journals.openedition.org/linx/775

DOI : 10.4000/linx.775

ISSN : 2118-9692

\section{Éditeur}

Presses universitaires de Paris Nanterre

\section{Édition imprimée}

Date de publication : 1 juin 1999

Pagination : 117-136

ISSN : 0246-8743

\section{Référence électronique}

Danielle Leeman, «L'unité lexicale dans la perspective harrissienne », Linx [En ligne], 40 | 1999, mis en ligne le 22 juin 2012, consulté le 06 mai 2019. URL : http://journals.openedition.org/linx/775 ; DOI : 10.4000/linx.775

Ce document a été généré automatiquement le 6 mai 2019.

Département de Sciences du langage, Université Paris Ouest 


\section{L'unité lexicale dans la perspective harrissienne}

Lexical Unity in a Harrisian Perspective

Danielle Leeman

\section{Introduction : le cadre théorique}

\subsection{Le principe de distribution}

1 Z. Harris (1951) ne conçoit pas d'emblée la langue comme un objet mental, donc inaccessible à l'observation et ainsi uniquement à élaborer par hypothèse, mais d'abord comme un ensemble de "discours», de combinaisons construites selon un principe distributionnel: le postulat préalable est que les énoncés s'analysent en unités "discrètes", c'est-à-dire isolables et combinables de telle sorte que toutes les associations ne sont pas " équiprobables »; c'est cette non-équiprobabilité qui fonde la «redondance » caractéristique des langues ${ }^{1}$ permettant d'identifier et de classer les entités linguistiques ; autrement dit, c'est à partir des contraintes qui atteignent les cooccurrences - rendant les unes possibles et les autres impossibles - que l'on peut saisir l'identité d'une occurrence donnée. Chacune est ainsi identifiée selon son aptitude à entrer dans une unité plus vaste: le phonème n'est par conséquent pas caractérisé, comme dans la tradition européenne, par sa constitution (un ensemble de traits distinctifs) mais en tant qu'il entre dans telles combinaisons et non d'autres, et de même, dans ce contexte, une approche sémique ou atomiste du sens n'est pas envisageable pour rendre compte de la signification des unités dites significatives.

\section{2. Le statut du sens}

2 Le second principe guidant la méthodologie est que, la "redondance " provenant de l'institution par la langue de dépendances combinatoires qui lui sont propres, l'identification, le classement ou l'attribution d'une signification aux unités linguistiques 
ne peuvent se fonder sur un recours au sens entendu comme le rapport que l'on établit intuitivement entre une forme et le monde tel qu'il nous apparaît (critère référentialiste) ou tel que nous le concevons (critère conceptualiste). Une telle démarche ne pourrait d'ailleurs pas être adoptée de manière systématique : définir le morphème comme la plus petite unité douée de sens empêcherait ainsi d'identifier clure dans inclure, conclure, exclure comme morphème (on ne voit pas quelle notion lui faire correspondre pour justifier qu'il s'agit d'une forme minimale significative), ou à l'inverse obligerait à multiplier les homonymes, aboutissant à poser autant de morphèmes que l'on observe de sens par exemple pour le suffixe -iser dans neutraliser (= « rendre neutre »), minimiser (暍 "rendre minime», = "présenter comme minime»), utiliser (䔽 《rendre utile», 䜿 " présenter comme utile »). De même, rassembler des unités dans une classe sur le critère du sens amènerait à ranger fréquent et souvent dans la même catégorie (ils signifient tous deux « un grand nombre de fois ») mais fréquent et sale dans des catégories différentes - et donc conduirait à des formules structurales agrammaticales, autorisant aussi bien Il vient fréquent/souvent ou Cela est fréquent/souvent, ou inversement ne prédirait pas des phrases possibles.

3 L'intuition sémantique spontanée - en tant qu'elle fait correspondre une certaine forme à un certain objet ou événement réel ou conçu - ne coïncide d'ailleurs pas avec la signification telle qu'instituée par la langue ; ainsi mettre et poser seront définis par un dictionnaire (pour un premier sens) comme "faire occuper à une chose un endroit déterminé » (mettre/poser un vase sur la table), mais les emplois effectifs des deux verbes répondant à cette définition montrent qu'elle est inadéquate, supposant une relation paraphrastique entre (1.a) et (1.b) ou (1.a) et (1.c) :

\begin{tabular}{|l|l|l|}
\hline & (1.a) & Luc met son manteau \\
\hline (1.b) & Luc pose son manteau \\
\hline & $(1 . \mathrm{c})$ & Luc met son manteau sur le fauteuil \\
\hline & $(1 . \mathrm{d})$ & Luc pose son manteau sur le fauteuil \\
\hline
\end{tabular}

Or (1.a) implique que «l'endroit déterminé » soit la personne même de Luc, ce qui n'est pas le cas de (1.b), et fait que (1.a) et (1.c) n'évoquent pas la même situation, contrairement à (1.b) et (1.d).

On identifie donc le morphème non en tant qu'unité minimale significative - i.e. selon le critère qu'on peut attribuer un sens à telle séquence de phonèmes - mais selon les contraintes qui atteignent les combinaisons de phonèmes dans l'énoncé. Ainsi, on peut repérer que [a] et [y] relèvent de deux phonèmes au fait que si quelqu'un dit [an] et qu'on lui demande s'il a dit [an], il répondra que non: [a] et [y] commutent devant [n]; mais dans le contexte [tabl], je peux dire [yntabl] et non [antabl] ou dans le contexte [døz], je peux dire [døzan] et non [døzyn]; ici, [a] et [y] ne sont plus commutables, ce sont les suites [an] ou [yn] qui le sont avec d'autres séquences, respectivement avec [urs] ou [la] par exemple: [døzurs], [latabl]. Et de même, c'est leur substituabilité formelle dans un contexte donné (et non leur sens) qui fera ranger dans une même classe [yn] et [la], ou [an] et [urs]. 


\section{3. Sens et information}

6 Il y a une relation entre langue et (conceptualisation $\mathrm{du}$ ) monde: le sens, mais elle échappe à la grammaire ; en matière de signification, le domaine propre de cette dernière est ce que Z. Harris appelle "l'information $»^{2}$, c'est-à-dire la structuration du sens par l'organisation des formes dans la langue. Du fait que l'unité lexicale appartient à un système (un ensemble de dépendances) et que son identité est donc tributaire de celle de toutes les autres, elle ne peut être considérée abstraction faite de ses occurrences : au moins en première approximation, sa signification ne peut apparaître qu'en emploi et n'être appréhendée intuitivement qu'en termes de similitude et de différence (-iser dans utiliser n'a pas le même sens relativement à utile que -iser dans neutraliser par rapport à neutre) ; sa relation aux réalités mondaines ou aux concepts - en somme, sa référence est hors de champ : pour Z. Harris, la sémantique ne relève pas de la grammaire.

7 Ce n'est pas dire, comme certains l'ont affirmé, que la grammaire ainsi entendue ne s'occupe résolument pas de l'interprétation des mots et ne se fixe pour tâche que l'inventaire d'enchaînements de formes vidées de leur signification; cela signifie que le sens (tel que l'organise la langue) doit être construit à partir de l'observation du fonctionnement des formes. Ainsi, le fait que sauter puisse être mis en relation avec saut par le biais de faire un saut ou faire du saut (J. Giry-Schneider 1978) est l'indice qu'il a (au moins) deux interprétations, ce que corrobore la différence distributionnelle - face à (2.a, b), on n'a pas indifféremment (3.a, b) :

\begin{tabular}{|l|l|l|}
\hline & $(2 . a)$ & Max saute \\
\hline & $(2 . b)$ & Médor saute \\
\hline & $(3 . a)$ & Max fait un saut / fait du saut \\
\hline & $(3 . b)$ & Médor fait un saut / ?? fait du saut \\
\hline
\end{tabular}

De même, l'ambiguïté de (4.a) se repère au fait que la phrase peut être reliée à deux ensembles morphosyntaxiques paraphrastiques (4.b) et 4.c) :

\begin{tabular}{|c|c|c|}
\hline$(4 . a)$ & & Le patron augmente les ouvriers (J. Giry-Schneider 1994) \\
\hline \multirow[t]{4}{*}{ (4.b) } & $=$ & Le patron donne une augmentation aux ouvriers \\
\hline & $=$ & Les ouvriers ont une augmentation \\
\hline & 砶 & Les ouvriers augmentent \\
\hline & 医 & Les ouvriers sont en augmentation \\
\hline$(4 . c)$ & $=$ & Les ouvriers augmentent \\
\hline & $=$ & Les ouvriers sont en augmentation \\
\hline
\end{tabular}




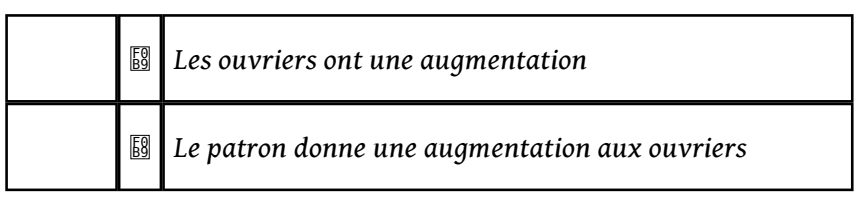

Concrètes, se prêtant donc à l'observation et à la manipulation, les formes sont le moyen d'accéder au sens (linguistique), tandis que le sens (l'idée que l'on a des choses) ne permet pas en soi de découvrir ou de décrire les formes parce que rien ne garantit qu'il s'agisse du sens tel que le conçoit la langue et donc tel qu'il est relié aux formes : si augmenter c'est « rendre plus grand» (Grand Larousse de la langue française), on ne peut pas pour autant dire La soupe augmente les enfants (ce que laisse pourtant supposer la définition sémantique et des formulations telles que Mange ta soupe, ça fait grandir); et inversement, on ne s'attend pas à pouvoir dire (4.a) puisqu'il ne s'y agit pas de rendre les ouvriers plus grands.

10 Le recours au sens dans la méthodologie est donc minimal, l'intuition s'exerçant simplement pour rassembler ou séparer des ensembles formels sur la base d'une similitude ou d'une différence, rassemblement ou séparation qui devront eux-mêmes se vérifier par leur rentabilité descriptive ; ainsi les récents travaux de l'équipe de Gaston Gross montrent-ils que l'on peut définir de vastes classes, homogènes sémantiquement du point de vue intuitif, à partir d'un faisceau de distributions (« les opérateurs appropriés ») et de transformations qui leur est spécifique (G. Gross 1994), par exemple dans les « humains » les « noms de professions » (menuisier, etc.), de " grades» (capitaine, etc.), de «relations» (frère, etc.) ou dans les «concrets» les «moyens de transport», les "vêtements", les «écrits" (D. Le Pesant 1994), etc. ${ }^{3}$. Pour une présentation des présupposés théoriques, de la méthodologie et des résultats, on se reportera à Langages $\mathrm{n}^{\circ}$ 131, coordonné par D. Le Pesant et M. Mathieu-Colas, Paris, Larousse, 1998.

\section{4. La dépendance de l'unité relativement à l'énoncé}

11 Si l'on ne peut pas définir le sens d'une unité lexicale considérée isolément, par un étiquetage venu de l'extérieur - c'est-à-dire attribué indépendamment du fonctionnement des formes linguistiques -, cela entraîne qu'on ne peut le saisir que comme relation : de même que l'appartenance de l'entité à telle ou telle catégorie ne peut être déterminée indépendamment de l'énoncé plus vaste où elle s'inscrit (puisque la classe est l'ensemble des éléments commutables en un point de l'axe syntagmatique), de même sa signification n'apparaît et ne peut être saisie qu'en contexte, à partir du moment où l'on pose en principe que la référence est extérieure à la langue.

12 A l'inverse, J.-C. Milner (1989) considère qu'« il est possible d'établir les propriétés d'un terme hors emploi » (p. 315), ce dont témoigne le fait que « le sujet sait reconnaitre hors contexte (à supposer bien évidemment qu'il sache la langue) qu'un terme est, par exemple, un Nom ou un Verbe (ou éventuellement les deux), qu'il a telle forme phonologique, qu'il a tel sens lexical, etc. » (p. 316); cependant, en ce qui concerne la reconnaissance de la catégorie (Nom ou Verbe), je dirais que cela suppose que le locuteur sache la grammaire, plutôt que la langue (or la grammaire s'appuie précisément sur les commutations en contexte pour dégager ses "parties du discours»); quant au sens lexical tel qu'il est spontanément attribué, rien ne dit qu'il ne sélectionne pas implicitement un certain environnement (c'est le cas de « rendre grand » pour augmenter 
ou de "faire occuper à une chose un endroit déterminé " pour mettre) : à la question "Que veut dire sauter?", celui qui répond « s'enlever du sol par la force des muscles puis revenir à sa place » n'a sans doute pas pensé au parachutiste qui tombe du ciel, au candidat au suicide qui se jette du dixième étage ni au chien qui vous saute à la gorge (et s'y tient)! De surcroît, cette réponse " hors contexte » est le résultat d'une acquisition qui s'opère le plus généralement via des discours - qu'on pense aux échanges entre parents et enfants, cf. C. Martinot 1998 - plutôt que par une mise en relation entre un mot et « un objet du monde $»^{4}$; je ne vois d'ailleurs pas comment une telle correspondance pourrait s'établir pour saisir le sens de aller ou recevoir dans $T u$ vas recevoir une gifle, de prendre et bon dans On va prendre un bon bain, ou de demain, passer, matinée dans Demain, tu passes la matinée chez la nourrice ${ }^{5}$ (D. Leeman 1997).

La théorie de la langue comme système suppose donc (pour Z. Harris) une interdépendance des formes, une interdépendance des significations et une interdépendance des unes par rapport aux autres (et réciproquement) car, d'une part, l'interdépendance des formes ne peut s'appréhender qu'en recourant à l'interdépendance des significations (ainsi le regroupement des formes en (4.b) distingué de celui de (4.c) s'opère selon le sentiment intuitif que l'on a deux ensembles paraphrastiques); et, d'autre part, l'interdépendance des significations ne nous apparaît jamais qu'à travers les formes, que ces dernières soient orales, écrites, gestuelles, etc. - une forme peut ne pas être manifeste en surface, son effacement matériel n'en laisse pas moins une trace sémantique; ainsi l'ambiguïté de (4.a) peut-elle être décrite comme le résultat de la suppression des "noms appropriés " ${ }^{6}$ salaire ou nombre: Le patron augmente (le salaire de) les ouvriers, Le patron augmente (le nombre de) les ouvriers.

Pour résumer, Z. Harris ne nie pas la corrélation possible entre les unités linguistiques et les « objets du monde ", mais il appartient au dictionnaire - et non à la grammaire - de l'établir : certes la langue parle du monde (i.e. des réalités en rapport avec l'expérience des individus dans une certaine culture), mais en tant qu'objet de la linguistique (le linguiste ne se donne pas comme objet le tout de la langue), elle est définie (restrictivement) comme un ensemble d'éléments fonctionnant sur le principe distributionnel, selon lequel ces éléments apparaissent de manière régulière dans des environnements caractérisables; il y a donc deux sémantiques : l'une qui décrit le sens (information) en tant qu'il obéit à ce principe - autrement dit le sens ainsi que l'organise la langue telle qu'on l'a définie -, l'autre qui décrit le sens (meaning) en tant qu'il échappe à ce principe - puisque l'on n'est plus dans le domaine de la structuration du sens par les formes - et réclame d'autres moyens d'investigation que la première (Z. Harris 1991) ${ }^{7}$.

Si l'on convient d'appeler « lexique » l'ensemble des unités linguistiques caractérisables selon le principe distributionnel dans la forme et le sens (première sémantique ci-dessus), et «Lexique » le domaine d'investigation qui a pour objet le lexique, alors le Lexique relève de la grammaire; on définira dans ce cadre l'unité lexicale comme membre du lexique passible d'un traitement grammatical (c'est-à-dire qui ne s'occupe pas de la référence).

\section{5. Lexème et morphème}

Dans le lexique tel qu'on l'a grossièrement défini - en première approximation, la liste des entrées d'un dictionnaire - se trouvent en fait deux sortes d'unité, traditionnellement étiquetées «lexèmes» et «morphèmes». On peut définir les premiers (ou "unités 
lexicales ») par le fait qu'ils sont responsables des échelles d'acceptabilité lors des commutations dans une catégorie donnée (A. Guillet 1986); soit par exemple la suite grammaticale $\mathrm{D}+\mathrm{N}+\mathrm{V}$, tous les membres de $\mathrm{N}$ ne sont pas indifféremment associables à tous les membres de $\mathrm{V}$; ainsi, miauler a une probabilité d'occurrence plus grande après chat qu'après chien, et c'est l'inverse pour aboyer :

\begin{tabular}{|l|l|l|l|}
\hline & (5.a) & & Le chat miaule \\
\hline & & & Le chien aboie \\
\hline & $(5 . \mathrm{b})$ & $? ?$ & Le chat aboie \\
\hline & & $? ?$ & Le chien miaule \\
\hline
\end{tabular}

Les seconds (ou "unités grammaticales») sont des outils syntaxiques en tant qu'ils permettent aux premiers de fonctionner dans une phrase; en l'occurrence, l'article permet au nom d'être sujet, et sans désinence de conjugaison, le verbe ne permet pas à la séquence d'être reconnue comme phrase. On ne peut cependant réduire l'unité grammaticale à une forme dotée d'une valeur fonctionnelle, car il existe des classes de morphèmes et, là comme ailleurs, la substitution de l'un à l'autre n'est pas indifférente : le et un introduisent tous deux un nom, la désinence de présent commute avec celles de futur ou d'imparfait, -age et -ment servent l'un comme l'autre à construire des noms ( nettoyage, nettoiement), etc.

$\mathrm{Si}$, donc, la syntaxe est dans les contraintes formelles de combinaison du lexique dans la phrase, associations qui mettent en jeu des lexèmes et des entités proprement syntaxiques (les places relatives, les morphèmes - que décrit la Syntaxe, comprise comme le domaine de la grammaire qui rend compte de la syntaxe), ces dernières ne sont pas seulement fonctionnelles (i.e. uniquement responsables de la grammaticalité): on admettra que le sens grammatical (ou syntaxique) contribue à la signification globale en donnant à voir de quelque manière le sens lexical, il constitue en quelque sorte une modalité de ce dernier ; ainsi, dans verre à vin et verre de vin, les prépositions introduisent deux modes de la relation entre verre et vin (P. Cadiot 1993a, 1993b), de même que le et un présentent différemment chat ou -age et -ment ce que véhicule la racine verbale.

En conclusion, la grammaire décrit la langue - ici sous-ensembledu tout de la langue, délimité par le principe de distribution - en tant que forme instituant du sens; sa méthode se fonde sur les formes, seules observables, analysées à l'aide d'une intuition de similitude et de différence sémantique.

Construire une phrase, c'est articuler entre elles (syntaxe) des unités lexicales (lexique). La grammaire, qui décrit la phrase, inclut donc une Syntaxe (qui rend compte de la syntaxe) et un Lexique (qui rend compte du lexique). Le principe de distribution reposant sur l'hypothèse de l'interdépendance des entités linguistiques (lexique et syntaxe, et réciproquement), la question se pose de celle du Lexique par rapport à la Syntaxe (et vice versa). 


\section{Syntaxe et Lexique / Lexique et Syntaxe : autonomie ou dépendance?}

\section{1. La Syntaxe est-elle dépendante du Lexique?}

Si la Syntaxe dépend du Lexique, alors on s'attend à ce que (a) les unités syntaxiques correspondent aux unités lexicales, et à ce que (b) la définition des relations syntaxiques soit tributaire de celle des relations entre entités lexicales. Mais ce n'est pas ce que l'on observe systématiquement :

(a) Il existe en effet des catégories syntaxiques qui ne sont pas des unités lexicales, comme les pronoms il, ce, qui, la conjonction que, les affixes -age, -ment - bref: les morphèmes. J.-C. Milner (op. cit.) avance l'exemple des interjections pour illustrer le cas où une forme n'aurait ni sens lexical ni appartenance catégorielle, car « il va de soi que la catégorie interjection est en réalité une non-catégorie » (p. 353); je dirais plutôt que le nom même de la catégorie signale qu'il s'agit de termes auxquels il est difficile d'assigner une fonction dans la structure phrastique telle qu'elle est habituellement définie, c'est-àdire hors énonciation, en en supprimant en particulier les témoignages de l'affectivité du sujet parlant (il en va de même des expressions manifestant une «non-coïncidence du dire " (J. Authier 1992) comme disons dans Je viendrai disons lundi); le fait que les interjections entrent dans les dictionnaires et y reçoivent une définition montre par ailleurs que ces mots ne sont pas dénués de sens (J. Tardieu 1951).

Une deuxième raison en faveur de l'indépendance de la Syntaxe par rapport cette fois au lexique est qu'elle définit ses catégories comme des classes de substitution sans se fonder sur le sens (lexical) des mots; c'est pourquoi, du reste, réduire la description grammaticale à celle de structures (donc aux résultats de la Syntaxe) empêche de séparer dans les phrases celles qui sont acceptables de celles qui le sont moins ou qui ne le sont pas : ainsi, la construction $\mathrm{D}+\mathrm{N}+\mathrm{V}$ (syntaxiquement recevable, par opposition à $\mathrm{N}+\mathrm{D}+\mathrm{V}$ en français), autorise aussi bien Le garçon chante que Du garçon chante, Le garçon germe, Le noyau chante, etc. Pour Z. Harris, la grammaire doit aussi rendre compte des phrases, c'est pourquoi elle inclut le Lexique.

(b) De même, la Syntaxe définit ses relations sans se fonder sur l'identité lexicale (vue comme le terme en tant qu'il est doté d'une catégorie et d'un certain sens, celui-ci défini par telles possibilités et impossibilités d'association avec les autres termes). Ainsi, dans la grammaire harrissienne, distingue-t-on entre " argument » et "opérateur ${ }^{8}$, le premier étant le support de la prédication que constitue le second: dans la phrase L'enfant dormait, enfant est l'argument de dormir et dormir l'argument de -ait; l'argument peut donc être (en l'occurrence) un nom ou un verbe et l'opérateur un verbe (dormir pour enfant) ou un affixe (-ait pour dormir) ; dans Tu es un enfant, enfant est opérateur. Les rôles syntaxiques reçoivent donc une définition qui traverse aussi bien les mots susceptibles de les occuper que les classes auxquelles ils appartiennent.

\section{2. Le Lexique est-il dépendant de la Syntaxe?}

Parallèlement aux critères précédents (2. 1.), si le Lexique dépend de la Syntaxe, alors on s'attend à ce que (a) les unités lexicales soient toutes aussi des unités syntaxiques, et à ce 
que (b) la définition des relations entre les unités lexicales inclue celle des relations syntaxiques. Il semble que ce soit le cas :

a) Il n'y a pas de lexème qui ne serait pas doté d'une appartenance catégorielle: toute unité lexicale est donc aussi une unité syntaxique (alors que l'inverse n'est pas vrai, ainsi qu'on l'a vu ci-dessus). De plus, on peut admettre avec Z. Harris que l'appartenance catégorielle influe sur le sens lexical (nous avons avancé plus haut que le sens grammatical n'est pas seulement fonctionnel, constituant une modalité du sens lexical) en ceci que la classe détermine un point commun (sémantique) à l'ensemble de ses membres $^{9}$ :

" ... beaucoup de classes de morphèmes (ou, dans certains cas, des sous-classes) ont un sens commun à la classe. Dans bon nombre de langues, on voit que des classes déterminées distributionnellement (de morphèmes ou de séquences de morphèmes) ont des sens que l'on peut grossièrement identifier par "nom", « verbe », « préposition », etc. » (Z. Harris 1951, p. 252 - je traduis).

De fait, la signification se présente différemment selon que la notion se moule par exemple dans un nom (comme bonté) ou un adjectif (comme bon) puisque les deux n'ont pas les mêmes propriétés distributionnelles; l'adjectif peut concerner aussi bien homme que pain :

\begin{tabular}{|l|l|l|}
\hline & (6.a) & Cet homme est bon \\
\hline & $(6 . \mathrm{b})$ & Ce pain est bon \\
\hline
\end{tabular}

mais on n'a pas parallèlement :

\begin{tabular}{|l|l|l|}
\hline$\left(6 . a^{\prime}\right)$ & La bonté de cet homme \\
\hline & $\left(6 . b^{\prime}\right) ? ?$ & La bonté de ce pain \\
\hline
\end{tabular}

De même, la transitivité directe du verbe admirer s'oppose à la démarcation qu'introduit la préposition avec le nom admiration ${ }^{10}$ :

\begin{tabular}{|l|l|l|}
\hline & (7.a) & Paul admire ce travail \\
\hline (7.b) & Paul a de l'admiration pour ce travail \\
\hline
\end{tabular}

et les deux formulations se distinguent par rapport à l'impératif par exemple :

\begin{tabular}{|l|l|l|l|}
\hline & $(8 . \mathrm{a})$ & & Admire! \\
\hline & & & Admire le travail! \\
\hline & & & Admire (comme c'est beau + si c'est beau)! \\
\hline & $(8.6)$ & $? ?$ & Aie de l'admiration! \\
\hline
\end{tabular}




\begin{tabular}{|l|l|l|l|}
\hline & & $?$ & Aie de l'admiration pour ce travail ! \\
\hline & & $*$ & Aie de l'admiration (comme c'est beau + si c'est beau)! \\
\hline
\end{tabular}

L'hypothèse d'une dépendance entre catégorisation syntaxique et signification de l'unité lexicale, telle que la première a une incidence sur la seconde, serait contredite par le cas où deux unités de même sens appartiendraient à des catégories différentes : J.-C. Milner ( op. cit. p. 348) cite pour l'illustrer l'exemple anglais de fish (nom dans the fish et verbe dans to fish); mes faibles compétences dans cette langue m'interdisent d'apprécier les similitudes entre les deux (qui seraient exactes si l'on met à part ce qu'apporte grammaticalement le fait même d'être un nom ou un verbe). Peut-être un équivalent français pourrait-il être rire ou sourire, cependant on peut rire (ou sourire) d'un comportement, d'une idée, d'une réponse tandis que le génitif du nom ne peut pas être objectif (le rire de Paul vs * le rire de ce comportement). La pêche n'est pas non plus la seule version nominale de pêcher, pouvant désigner non seulement «l'action de prendre du poisson » (comme le verbe) mais aussi son « résultat ».

b) Cependant la Syntaxe ne peut être réduite à la catégorisation, ni à la description de la phrase comme combinaison (dans un certain ordre) de catégories. On observe en effet d'abord que certaines structures peuvent être mises en rapport du fait que les relations distributionnelles sont identiques bien que la succession des classes diffère ${ }^{11}$ (cf. (9.a, b) emprunté à Z. Harris 1968). On constate ensuite inversement qu'une même formule linéaire peut cacher des connexions syntaxiques diverses entre les catégories qui la composent (cf. (10.a, b) extrait de Z. Harris 1968) ${ }^{12}$.

\begin{tabular}{|l|l|l|l|}
\hline & (9.a) & & A dog chewed a bone \\
\hline & & ?? & A book chewed a cup \\
\hline \hline (9.b) & & A bone was chewed by a dog \\
\hline & & ?? & A cup was chewed by a book \\
\hline$(10 . \mathrm{a})$ & & It was sent by the senator from Ohio \\
\hline & $=$ & The senator sent it from Ohio \\
\hline$(10 . \mathrm{b})$ & & It was sent by the senator from Ohio \\
\hline & & $=$ & The senator is from Ohio \\
\hline
\end{tabular}

31 Il s'agit alors de savoir s'il y a un lien de dépendance entre l'unité lexicale et la corrélation syntaxique telle que cette dernière détermine le choix du lexème. Des exemples comme (11.a, b) montrent que c'est le cas (M. Gross 1975) :

(11.a) Ce problème concerne Marie 


\begin{tabular}{|l|l|l|}
\hline & & Marie est concernée par ce problème \\
\hline & $(11 . b)$ & Ce problème regarde Marie \\
\hline & & *Marie est regardée par ce problème \\
\hline
\end{tabular}

puisque, toutes choses égales par ailleurs, la construction passive permet le choix de concerner mais interdit celui de regarder. Dit autrement, l'identité du verbe inclut dans sa définition qu'il entre dans tel type de structure et non dans tel autre : la description des unités par le Lexique doit donc inclure une référence aux résultats de la Syntaxe.

J.-C. Milner (op. cit.) conclut à l'inverse à l'indépendance réciproque du Lexique et de la Syntaxe à partir des exemples $(12 . \mathrm{a}, \mathrm{b})$ qui montrent que la variation sémantique peut être due au lexique seul et (13.a) où elle provient uniquement de la syntaxe (op. cit. p. 289) :

\begin{tabular}{|l|l|l|}
\hline & $(12 . \mathrm{a})$ & Le silence éternel de ces espaces infinis m'effraie (Pascal) \\
\hline & $(12 . \mathrm{b})$ & Le bavardage intermittent de nos petites sociétés me rassure (d'après Valéry) \\
\hline & $(13 . \mathrm{a})$ & Je lui propose de venir demain \\
\hline
\end{tabular}

Il remarque (note 8, p. 297) que le lexique et la syntaxe contribuent chacun à sa manière à la construction du sens, ce qui corrobore l'hypothèse qu'ils sont autonomes; à la modification sémantique telle qu'illustrée en (12.a, b) s'oppose en effet celle qui concerne $(14 . a, b)$ :

\begin{tabular}{|l|l|l|}
\hline & $(14 . \mathrm{a})$ & Sylvie adore Renoir \\
\hline \hline & $(14 . \mathrm{b})$ & Renoir, Sylvie adore \\
\hline
\end{tabular}

où la différence de linéarisation (les places occupées par les constituants) ne change pas la relation entre les « sites », au contraire de (14.c) :

(14.c) Renoir adore Sylvie

où Renoir et Sylvie n'ont plus la même fonction syntaxique.

Il me semble que la démonstration n'est pas totalement convaincante car d'une part, ce qui permet de dire que (12.a) et (12.b) ne varient qu'en signification, c'est le choix des mots; une sélection lexicale différente aurait pu aboutir à la conclusion que les phrases varient également dans la syntaxe :

(12.c) Le garçon silencieux de ses yeux moqueurs me provoque 
Et d'autre part, de même, l'ambiguïté syntaxique de (13.a) disparait si l'on remplace proposer par interdire ou promettre :

\begin{tabular}{|l|l|l|}
\hline (13.b) & Je lui interdis de venir demain \\
\hline (13.c) & Je lui promets de venir demain \\
\hline
\end{tabular}

comme s'estompe la différenciation qui s'opère quand des lexèmes identiques occupent des sites différents (exemples (3.a, b) de J.-C. Milner, op. cit., p. 297) :

\begin{tabular}{|l|l|l|}
\hline & $(14 . \mathrm{d})$ & Sylvie aime Bruno \\
\hline & $(14 . \mathrm{e})$ & Bruno aime Sylvie \\
\hline
\end{tabular}

lorsque l'on change les unités lexicales des noms et du verbe :

\begin{tabular}{|l|l|l|}
\hline & $(15 . \mathrm{a})$ & Ce peintre est Renoir \\
\hline & $(15 . \mathrm{b})$ & Renoir est ce peintre \\
\hline
\end{tabular}

Tout ce que l'on peut dire, au vu des exemples (12) à (15), c'est qu'il y en a autant qui illustrent la thèse de l'autonomie que celle de la dépendance. On avancera donc plutôt l'idée suivante : la Syntaxe définit des combinaisons formelles (i.e. en termes de catégories et de sites) en raisonnant sur quelques exemples concrets, si bien que la généralité de la description n'est pas garantie (on n'a pas vérifié qu'elle vaut pour toutes les associations lexicales) ${ }^{13}$. De fait, les structures ou relations ne concernent pas tous les termes susceptibles d'appartenir aux catégories mises en jeu : de même que la formule $\mathrm{D}+\mathrm{N}+\mathrm{V}$ correspond telle quelle aussi bien à Le garçon chante qu'à Du garçon chante ou Le garçon germe, le passage de l'actif au passif est possible pour concerner et non pour regarder en (11) ou le déplacement frontal acceptable pour Renoir en (14.a, b) mais non pour Médor en (14.f) :

\begin{tabular}{|l|l|l|l|}
\hline & $(14 . \mathrm{f})$ & & Sylvie adore Médor \\
\hline & & $? ?$ & Médor, Sylvie adore \\
\hline
\end{tabular}

Cela n'invalide pas le bien-fondé de la description syntaxique mais oblige en retour à spécifier les unités lexicales (si du moins, comme Z. Harris, on veut rendre compte des phrases et non seulement des structures). On retrouve donc la thèse de la dépendance du Lexique relativement à la Syntaxe: la caractérisation des unités lexicales inclut leur définition catégorielle d'une part et leurs possibilités syntaxiques d'autre part, déterminées par la vérification que tel mot particulier admet telle combinaison où entre la catégorie à laquelle il appartient. Ainsi, quoique nom propre comme Renoir, Médor exclut la relation de type (14.a, b), ce qui alerte sur la nécessité de préciser la définition 
des termes entrant dans la classe des noms propres; ce serait vrai aussi des noms communs, des déterminants et des verbes, comme le montrent (16.b, c, d) comparés à (16.a) :

\begin{tabular}{|l|l|l|l|}
\hline & $(16 . \mathrm{a})$ & & Le chocolat, j'adore \\
\hline & $(16 . \mathrm{b})$ & ?? & Le boulanger, j'adore \\
\hline & (16.c) & ?? & Ton chocolat, j'adore \\
\hline & (16.d) & ?? & Le chocolat, je mange \\
\hline
\end{tabular}

La généralité des règles de la Syntaxe passe par la spécification de sous-classes lexicales, ce que l'on peut formuler : les règles syntaxiques imposent leur loi aux termes en ceci que seuls certains d'entre eux sont admis à figurer dans les structures.

En résumé, l'identité lexicale doit s'appréhender d'une part par le biais de ses relations distributionnelles (Le problème regarde/concerne Marie est une combinaison plus probable que Ce garçon regarde/concerne Marie ${ }^{14}$ ) et d'autre part selon ses combinaisons syntaxiques (dans l'emploi défini précédemment, concerner mais non regarder admet la transformation passive), celles-ci présupposant celles-là puisque les relations lexicales sont testées dans un certain cadre syntaxique.

\section{Le sens des unités grammaticales}

40 S'il appartient donc au Lexique d'intégrer dans sa description du lexique les possibilités syntaxiques des unités lexicales, la difficulté est de les interpréter de manière à en faire son profit dans la caractérisation du sens du lexème: quelle valeur attribuer au passif comparé à l'actif, et comment par elle spécifier l'identité de regarder (dans cet emploi), qui exclut le passif, par rapport à concerner, qui l'admet ? Le sens de la structure étant $a$ priori moins accessible que celui du terme, on est tenté de partir de ce dernier (appréhendé intuitivement) pour expliquer l'impossibilité ou la compatibilité, mais les études systématiques montrent qu'il n'y a là rien de facile (C. Leclère 1993) - ce qui est logique si l'on admet que le sens attribué intuitivement ne correspond pas au sens tel qu'institué par les formes. Ce qui reste en suspens, par conséquent, c'est la contribution du sens grammatical (celui des morphèmes ou de la structure) au sens lexical - et donc, préalablement, sa définition même.

41 En l'occurrence, le cadre harrissien ne nous est d'aucune aide : on peut caractériser une classe $X$ (étiquetée par commodité pédagogique «noms de vêtements») par des opérateurs tels que mettre, enlever, en, etc. : mettre, enlever une robe; être en robe (D. Le Pesant et M. Mathieu-Colas, op. cit.), classe à laquelle n'appartient pas mouchoir :

\begin{tabular}{|l|l|l|}
\hline & $(17 . a)$ & $*$ Il met/enlève un mouchoir \\
\hline & $(17 . b)$ & *Il est en mouchoir \\
\hline
\end{tabular}


mais par rapport à laquelle des noms comme vêtement ou habits posent un problème, puisque si l'on peut mettre ou enlever un vêtement, des habits, la construction avec en n'apparait pas possible (D. Leeman 1996b) :

(18.a) *Eve est en vêtement, en habits

à moins de qualifier le nom :

(18.b) Eve est en vêtement de deuil, en habits des dimanches

On peut naturellement conclure à l'existence de sous-classes (ainsi les «moyens de transport » recouvrent-ils les moyens « terrestres », « maritimes », « aériens, etc. - cf. D. Le Pesant et M. Mathieu-Colas op. cit.), mais la logique de la démarche (fondée, rappelonsle, sur l'interdépendance de la forme et du sens) est plutôt de chercher à savoir pourquoi en établit une telle partition, autrement dit quelle est la particularité sémantique que pointe pour le nom sa compabibilité ou incompatibilité avec en.

Pour ce faire, il me semble qu'il faut chercher à élaborer le signifié de la préposition en tâchant d'interpréter ses associations avec tels et non tels autres membres des divers paradigmes qu'elle peut introduire : les moyens de déplacement (en voiture mais non * en cheval), les sentiments (en joie mais non * en félicité), les lieux (en cuisine mais non * en salon ), etc. On peut avancer des hypothèses particulières successives (qu'il s'agira ensuite de synthétiser): en est reliable au "construit» (la voiture mais non le cheval), au " manifesté » (on peut sauter ou pousser des cris de joie mais non de félicité), au " statut » (la cuisine est un lieu lié à une activité susceptible de définir un rôle : dans un restaurant, il y a ceux qui sont en cuisine et ceux qui sont en salle), etc.

Les tendances communes dégageables de ces diverses observation aboutissent à l'hypothèse que en introduit un jugement qui a trait à l'extérieur de l'entité jugée (et non à sa nature): on peut être naturellement d'une totale incapacité mais ce que dit être en incapacité n'a pas trait à cette qualité inhérente; de même Paul est en femme est acceptable (en femme étant interprété comme un déguisement de Paul) mais non ?? Paul est en homme (à moins d'interpréter Paul comme le nom d'une femme). L'expression décrit un état résultatif donnant lieu à une caractérisation établie à partir de certains critères, d'une norme conventionnelle : la joie se repère à telle manifestation, la cuisine à telle activité, la femme à telle apparence ${ }^{15}$, etc.

On peut par là expliquer que les noms hyperonymes ne soient guère compatibles avec en (?? aller quelque part en véhicule) : en tant que mots génériques, ils ne possèdent pas les traits particuliers à partir desquels justifier la caractérisation par en; on rend compte ainsi de (18) :

\begin{tabular}{|l|l|l|}
\hline & $(18 . a) *$ & Eve est en vêtement, en habits \\
\hline & $(18 . b)$ & Eve est en vêtement de deuil, en habits des dimanches \\
\hline
\end{tabular}

(pour des propositions et un exemple détaillés, cf. D. Leeman 1998). 
En résumé, on est dans l'obligation de devoir construire (par hypothèse) le signifié des unités grammaticales, ce que l'on ne peut faire qu'à travers l'observation de leurs formes (les possibilités et contraintes morphologiques, distributionnelles et syntaxiques) puisque nous ne pouvons les mettre directement en correspondance avec (un concept issu de) notre expérience du monde. Cette situation confirme les présupposés théoriques harrissiens concernant le sens, à moins de supposer que la langue renferme deux systèmes étanches d'unités : d'un côté les lexèmes (dont on pourrait définir l'identité selon des critères référentiels ou conceptuels), et de l'autre les morphèmes (dont la caractérisation ne peut s'obtenir que par un travail purement linguistique) ${ }^{16}$.

\section{BIBLIOGRAPHIE}

AUROUX, S. (1998) «Les enjeux de la linguistique de terrain », Langages n 129, Paris, Larousse.

AUTHIER, J. (1992) Les non-coïncidences du dire et leur représentation méta-énonciative. Etude linguistique et discursive de la modélisation autonymique, Thèse de l'université de Paris VIII.

CADIOT, P. (1987) De la grammaire à la cognition. La préposition « pour », Paris, Editions du CNRS.

CADIOT, P. (1993a) «À entre deux noms : vers la composition nominale », Lexique $\mathrm{n}^{\circ} 11$, Presses universitaires de Lille.

CADIOT, P. (1993b) « De et deux de ses concurrents : avec et à », Langages n 110, Paris, Larousse.

DALADIER, A. (1990) éd. Les grammaires de Harris et leurs questions, Langages n 99, Paris, Larousse.

DUBOIS, J. (1979) « Annexe grammaticale », Dictionnaire du français langue étrangère, Paris,

Larousse.

DUBOIS, J. et al. (1994) Dictionnaire de linguistique et des sciences du langage, Paris, Larousse.

GIRY-SCHNEIDER, J. (1978) Les nominalisations en français, Genève-Paris, Droz.

GIRY-SCHNEIDER, J. (1994) « Sélection et sémantique, problèmes et modèles », Langages n 115, Paris, Larousse.

GROSS, G. (1993) éd. Sur le passif, Langages n 109, Paris, Larousse.

GROSS, G. (1994) « Classes d'objets et description des verbes », Langages n 115, Paris, Larousse.

GROSS, M. (1975) Méthodes en syntaxe, Paris, Hermann.

GUILLET, A. (1986) « Représentation des distributions dans un Lexique-grammaire », Langue française $n^{\circ} 69$, Paris, Larousse.

HARRIS, Z. (1951) Structural Linguistics, Chicago \& London, The University of Chicago Press.

HARRIS, Z. (1954 trad. 1970) « La structure distributionnelle », Langages n² 20, Paris, Larousse.

HARRIS, Z. (1968 trad. 1971) Structures mathématiques du langage, Paris, Dunod.

HARRIS, Z. (1976) Notes du Cours de syntaxe, Paris, Le Seuil.

HARRIS, Z. (1982) A Grammar of English on Mathematical Principles, New-York, John Wiley \& Sons. 
HARRIS, Z. (1988) Language and Information, New-York, Columbia University Press.

HARRIS, Z. (1991) A Theory of Language and Information, Oxford, Clarendon Press.

LANGACKER, R. (1987 trad. 1991) « Noms et verbes », Communications n53, Paris, Le Seuil.

LAKOFF, G. (1986) « Cognitive Semantics », Versus 44/45, Milan, Bompiani.

LECLÈRE, C. (1993) « Classes de constructions directes sans passif », in Gross, G. éd.

LEEMAN, D. (1996a) « Le sens et l'information chez Harris », Linx (numéro spécial 8 Du dire et du discours), Centre de Recherches linguistiques de Paris X.

LEEMAN, D. (1996b) « Vêture, coiffure, chaussures et autres... coquetteries », Hommages à JeanClaude Coquet, Paris, L'harmattan.

LEEMAN, D. (1997) « Définir une préposition : hypothèses et perplexités », Revue de Sémantique et de Pragmatique ${ }^{\circ} 2$, Presses de l'Université d'Orléans.

LEEMAN, D. (1998) Les circonstants en question(s), Paris, Kimé.

LE PESANT, D. (1994) « Les compléments nominaux du verbe lire, une illustration de la notion de « classe d'objets » ", Langages $n^{\circ} 115$, Paris, Larousse.

LE PESANT, D. et MATHIEU-COLAS, M. (1998) éds. Les classes d'objets, Langages n 131, Paris, Larousse.

MARTINOT, C. (1998) éd. L'acquisition du français langue maternelle, Langue française $\mathrm{n}^{\circ} 118$, Paris, Larousse.

MILNER, J.-C. (1989) Introduction à une science du langage, Paris, Le Seuil.

PICABIA, L. et Zribi-Hertz, A. (1981) Découvrir la grammaire française, Paris, CEDIC (distribué par Nathan).

TARDIEU, J. (1951) Un mot pour un autre, Paris, Gallimard NRF.

\section{NOTES}

1. Il s'agit ici de la «redondance» telle qu'on l'entend dans les théories de l'information des années quarante. Pour une définition très claire, cf. J. Dubois et al. (1994) pp. 400 sqq.

2. Cf. Z. Harris 1988, 1991 ; T. Ryckman 1990 ; pour une analyse de l'émergence de cette notion, D. Leeman 1996.

3. J. Dubois, dans son « Annexe grammaticale » au Dictionnaire du français langue étrangère, tome II (Paris, Larousse, 1979), avait jeté les bases d'un tel classement, dont les données sont incluses dans les définitions des entrées elles-mêmes.

4. Si l'on admet que la «molécule » la table est formée des « atomes" la et table, «on peut alors considérer que les propriétés pertinentes de l'atome table fonctionnent comme autant de conditions que doit remplir un objet du monde quelconque pour pouvoir être, éventuellement, désigné par une molécule dont table sera une partie » (J.-C. Milner, op. cit., p. 336).

5. «Jespersen cite l'exemple du mot groenlandais panygmah traduit dans un dictionnaire bilingue de 1587 par « aiguille ». On sait aujourd'hui que ce mot correspond à " celle de ma fille ». On imagine bien la situation de terrain: le traducteur aura pointé une aiguille du doigt et l'indigène aura compris qu'il voulait savoir à qui cette aiguille appartenait » (S. Auroux 1998, note 6 p. 93). J.-C. Milner se situe en l'occurrence dans le paradigme « objectiviste » contre lequel s'est élaborée la théorie cognitiviste: la syntaxe est conçue comme la manipulation algorithmique de symboles dont le sens (qui n'intervient pas dans la définition des règles) est 
dans leur corrélation avec des ensembles (définis en intension) des objets du monde. G. Lakoff (1986) montre que ce paradigme échoue des deux points de vue empirique et logique.

6. Le nom dit "approprié » est hautement probable dans son contexte et pour cette raison susceptible d'être effacé sans que cela affecte notablement l'interprétation ; ainsi, arrivée est un complément approprié pour attendre dans Max attend (l'arrivée de) Marie (Z. Harris 1982).

7. Le meaning me paraît relever de ce que J.-C. Milner (op. cit.) considère être les «propriétés absolues " ou "propriétés intrinsèques » du terme (pp. 315 sqq.) ; l'information correspondrait alors à ses « propriétés relationnelles » ou «propriétés extrinsèques ».

8. Pour une présentation en français, cf. Z. Harris (1976), traduit et adapté par M. Gross.

9. R. Langacker (1987 trad. 1991), dans un cadre théorique différent, défend le même point de vue, mais seulement « pour les noms, les verbes et certaines sous-catégories majeures » (p. 103).

10. Sur la définition de pour, cf. P. Cadiot (1987).

11. Cette observation conduit $\mathrm{Z}$. Harris à introduire la notion de "transformation " pour relier dans la grammaire les structures entretenant ce rapport (Z. Harris 1954).

12. L'« analyse en constituants immédiats» permet de rendre compte de certaines des ambiguïtés ainsi créées (Z. Harris 1951).

13. L'objectif de M. Gross et de son équipe du LADL est au contraire de tester la validité d'une relation syntaxique (par exemple le passif) sur l'ensemble des termes a priori concernés (en l'occurrence les verbes transitifs à complément direct).

14. Où regarde = concerne. Exemple emprunté à L. Picabia et A. Zribi-Hertz (1981).

15. «L'ami n'a pas de caractéristiques extérieures visibles susceptibles de fonctionner comme une norme à partir de laquelle on pourrait reconnaître le déguisement » (D. Leeman 1996b) : * Paul est en ami. Mais on peut parler en ami, venir en ami, etc. c'est-à-dire adopter le comportement qui est celui qu'on associe normalement à quelqu'un jugé être un ami.

16. Je remercie pour leur relecture attentive C. Marque-Pucheu, S. Meleuc, G. Petit et pour son aide amicale Yves Abrioux.

\section{RÉSUMÉS}

On rappelle les principes théoriques généraux de Z. Harris (l'interdépendance des formes et des sens et le fait que ces derniers ne peuvent s'appréhender qu'à travers les formes instituées par la langue) et la méthodologie descriptive qui en découle, travail d'observation qui permet de mettre au jour l'information, le sens étant hors du champ de la grammaire. Nous posons qu'il faut aller plus loin et interpréter les unités grammaticales aussi bien que lexicales si l'on veut pouvoir avancer des hypothèses d'explication sur les possibilités et contraintes distributionnelles.

This paper begins with a summary of Z. Harris' general theoretical principles (interdependence of forms and meanings; the fact that the latter can only be apprchended through the forms instituted by language) and of the descriptive methodology deriving from them - a process of observation which makes it possible to bring "information " to light, whercas "meaning" remains outwith the field of grammar. We suggest that it is necessary to go further and to interpret the constraints observed, in order to be able to put forward explanations regarding the distributive bchaviour of lexical units. 


\section{AUTEUR}

\section{DANIELLE LEEMAN}

Université de Paris X - Nanterre et UMR 5610 (CNRS) 